\title{
On the Convergence of LHAM and its Application to Fractional Generalised Boussinesq Equations for Closed Form Solutions
}

\author{
S. O. Ajibola ${ }^{1, *}$, E. O. Oghre ${ }^{1}$, A. G. Ariwayo ${ }^{2}$ and P. O. Olatunji ${ }^{2}$ \\ ${ }^{1}$ Department of Mathematics, University of Benin, Nigeria \\ e-mail: ylang3472@gmail.com (S. O. Ajibola) \\ ${ }^{2}$ Department of Mathematical Sciences, Adekunle Ajasin University, Nigeria
}

\begin{abstract}
By fractional generalised Boussinesq equations we mean equations of the form

$$
\Delta \equiv D_{t}^{2 \alpha}-[\mathcal{N}(u)]_{x x}-u_{x x x x}=0,0<\alpha \leq 1,
$$

where $\mathcal{N}(u)$ is a differentiable function and $\mathcal{N}_{u u} \neq 0$ (to ensure nonlinearity). In this paper we lay emphasis on the cubic Boussinesq and Boussinesq-like equations of fractional order and we apply the Laplace homotopy analysis method (LHAM) for their rational and solitary wave solutions respectively. It is true that nonlinear fractional differential equations are often difficult to solve for their exact solutions and this single reason has prompted researchers over the years to come up with different methods and approach for their analytic approximate solutions. Most of these methods require huge computations which are sometimes complicated and a very good knowledge of computer aided softwares (CAS) are usually needed. To bridge this gap, we propose a method that requires no linearization, perturbation or any particularly restrictive assumption that can be easily used to solve strongly nonlinear fractional differential equations by hand and simple computer computations with a very quick run time. For the closed form solution, we set $\alpha=1$ for each of the solutions and our results coincides with those of others in the literature.
\end{abstract}

Received: April 16, 2021; Accepted: May 18, 2021

2020 Mathematics Subject Classification: 26A33, 35R11, 44A10.

Keywords and phrases: fractional calculus, Laplace homotopy analysis method, Boussinesq equations, closed form solution.

${ }^{*}$ Corresponding author 


\section{Introduction}

Late 1695, based on Leibniz's notation, L'Hôpital asked Leibniz what $\frac{d^{1 / 2} y}{d x^{1 / 2}}$ would be. This question led to what is known today as fractional calculus which have witnessed a lot of contributions even though in its early days it developed mainly as a pure mathematical field that only pure mathematicians find useful. Recent decades have seen a rise in texts [4, 19, 32, 33, 34] dedicated to this field of mathematics in the applied mathematical sense and in its use to model real life phenomena in biology and bioengineering [10, 59, plasma physics, chemistry, viscoelasticity [11, 66], viscoplasticity [41], fluid mechanics, continuum mechanics [2], engineering, finance, rheology, electromagnetic theory and electric networks [18, 57, probability [25] and so on. Generally, no known method exists for the exact solutions of fractional differential equations and that is why recent decades have seen "births" and use of different methods and approach for the approximate [and sometimes analytic approximate] solutions of fractional differential equations. Some numerical methods include:

1. finite difference methods [7, 38];

2. series approximation methods such differential transform method, Adomian decomposition method [31, 51, 64, 65, 68] developed in [13, 14], variational iteration method [73] developed by J.H. He in [27, 28], homotopy perturbation method (HPM) 61] developed in [26], homotopy analysis method (HAM) developed by Shijun Liao in [62 - interested reader can find the comparison between HPM and HAM in [43, 47].

3. finite element method [29, 69]; and

4. several other methods like the spectral method [70, meshless methods [3, 34, 40, 49, 50], operational matrix method [19], and so on.

The 2010s saw the use of some methods like Laplace homotopy analysis method [44, the Lie group method [58], the first integral method [42], inverse differential operational method [35], F-expansion method [71], M-Wright transforms [37, exponential differential operators [1], and so on. Truly, finding exact solutions 
to fractional differential equations is hard work and will still remain an active area of research. Of all these methods, we have chosen to apply LHAM to generalized Boussinesq equations [67] of fractional order. The LHAM, recently used in 63], is a tweaked or modified HAM which means that it falls in the category of series approximation methods. LHAM gives a high deformation equation in simple form and it helps to overcome the deficiencies that is caused by unsatisfied conditions in other techniques. Unlike other series approximation methods, the LHAM is not cumbersome, can be used in a direct way without the use of linearization, perturbation, or any restrictive assumption. Compared to the HAM, HPM and other series approximation methods, its computation size is small and it convergences rapidly.

Researchers in recent decades have extensively worked on nonlinear evoluton equations (NLEEs) with new direction related processes that has been actively developing in various areas of science and engineering because of its importance in fluid mechanics, mathematical-, physical- and chemical science. In mathematical physics, the analytical solutions of NLEEs are of fundamental importance as they are usually in the form of a mathematical function and from these functions the numerical value(s) of the dependent variable can be computed for any value of the independent variable. More reasons for prefering analytical solutions are: (say) our NLEE contains a parameter, an analytic solution of the equation will also contain a parameter of which the behaviour of the solution of the equation as the value of the parameter changes can be easily understood. Also, when we obtain analytical solution of a NLEE without its attached boundary conditions, the arbitrary constants in the solution are parameters in the solution. In engineering, for example, NLEEs have been used to interprete recession hydrographs from sloping aquifers [9] and some analytical and numerical solutions can be seen in [22, 23, 24]. Of the infinite possible solutions to NLEEs, some special form solutions may depend only on a single combination of variables such as solitons [56. Solitons, also known as solitary waves, are self-reinforcing wave packets that maintain their shape while they travel at constant speed [54, 55]. Solitons are caused by a cancelation of nonlinear and dispersive effects - the property of 
certain systems where the speed of the waves varies according to frequency - in a medium and they arise as the solutions of a widespread class of weakly nonlinear dispersive partial differential equations that describe physical systems [46, 17]. John Scott Russell (1808-1882) is the first person to scientifically describe the soliton phenomenon when he observed a solitary wave in the Union Canal in Scotland and then reproduced the phenomenon in a wave tank and called it "wave of translation" (commonly called solitary wave or soliton today) [30, 17]. Decades later Joseph Valentin Boussinesq (1842-1929) in [20, 21] responded to the observation by John Scott Russell of the wave of translation birthing the Boussinesq approximation, a very important equation in fluid mechanics.

In fluid mechanics, the Boussinesq approximation for water waves is an approximation valid for weakly nonlinear and fairly long waves. The equations now known as the Boussinesq equations are the equations (25) and (26) in Boussinesq's paper of 1872 where eq. (25) from [21] is the set of PDEs given as

$$
\begin{aligned}
\frac{\partial \eta}{\partial t}+\frac{\partial}{\partial x}\left[(h+\eta) u_{b}\right] & =\frac{1}{6} h^{3} \frac{\partial^{3} u_{b}}{\partial x^{3}}, \\
\frac{\partial u_{b}}{\partial t}+u_{b} \frac{\partial u_{b}}{\partial x}+g \frac{\partial \eta}{\partial x} & =\frac{1}{2} h^{2} \frac{\partial^{3} u_{b}}{\partial t \partial x^{2}}
\end{aligned}
$$

and eq. (26) [from [21]] under some additional approximations, but at the same accuracy, reduces the above equation to a single partial differential equation for the free surface elevation $\eta$ given as:

$$
\frac{\partial^{2} \eta}{\partial t^{2}}-g h \frac{\partial^{2} \eta}{\partial x^{2}}-g h \frac{\partial^{2}}{\partial x^{2}}\left(\frac{3}{2} \frac{\eta^{2}}{h}+\frac{1}{3} h^{2} \frac{\partial^{2} \eta}{\partial x^{2}}\right)=0 .
$$

Over the years a lot of research work has been done to improve, modify, extend and apply the Boussinesq equations [15, 16, 45, 52, which has resulted in an overwhelming number of mathematical models which are now referred to as Boussinesq equations. This, easily, can lead to confusion since what is referred to as the Boussinesq equation is just a variant thereof. Strictly speaking, the Boussinesq equation is the equation (1.1) and the set of equations that led to it hence for any variant it is appropriate to call them Boussinesq-type equations. 
All these variants and the original equation can be generalised by the form given by $[8,67$ as

$$
u_{x x x x}+[\mathcal{N}(u)]_{x x}=u_{t t} .
$$

The soliton equation 1.2 , when $\mathcal{N}(u)=\frac{1}{2} u^{2}$, had earlier been considered by Boussinesq to describe the propagation of long water waves [20, 21] in shallow water and since then it has been used to model many other physical phenomena in science and engineering (see [9] and references therein and other references cited herein).

\section{Preliminary Definitions and Theorems}

Some important definitions and theorems are stated in this section as preliminaries to main work [63]. Let $X$ be a measure space. Define $\mathcal{M}\left(\mathbb{R}^{+}\right)$as the space of all measurable functions from $X$ to $\mathbb{R}^{+}$.

Definition 2.1. A real function $f(t), t>0$, is said to be in the space $C_{\beta}, \beta \in \mathbb{R}$ if there exists a real number $p(>\beta)$, such that $f(t)=t^{p} f_{1}(t)$, where $f_{1}(t) \in C[0, \infty)$, and is said to be in the space $C_{\beta}^{m}$ if and only if $f^{(m)} \in C_{\beta}, m \in N$.

Definition 2.2. A sequence $\left\{f_{n}\right\}$ of real-valued functions is said to converge uniformly on $X$ to a function $f$ if for each $\epsilon>0$ there exists some $n_{0}$ (depending on $\epsilon$ ) such that $\left|f_{n}(x)-f(x)\right|<\epsilon$ for all $n \geq n_{0}$ and all $x \in X$.

Theorem 2.3. If $\left\{f_{n}\right\}_{0}^{\infty} \subset \mathcal{M}\left(\mathbb{R}^{+}\right)$and $\sum_{n=0}^{\infty} f_{n}$ converge uniformly to $f$, then $f=\sum_{n=0}^{\infty} f_{n}$.

Definition 2.4. Laplace transform of a function $f(t)$ is defined as the improper integral

$$
F(s)=\mathcal{L}\{f(t) ; s\}=\int_{0}^{\infty} e^{-s t} f(t) d t
$$

such that the integral converges and exist.

Theorem 2.5. For any functions $f(t)$ and $g(t)$ and constants $\alpha, \beta$ where $F(s)$ and $G(s)$ exist,

$$
\mathcal{L}\{\alpha f(t)+\beta g(t)\}=\alpha F(s)+\beta G(s) .
$$


Definition 2.6. The inverse Laplace transform is defined as

$$
f(t)=\mathcal{L}^{-1}\{F(s) ; t\}=\frac{1}{2 \pi i} \int_{c-i \infty}^{c+i \infty} e^{s t} F(s) d s, \quad c=\operatorname{Re}(s)>c_{0},
$$

and consequently $\mathcal{L}^{-1}(\mathcal{L}(f))=f$ and $\mathcal{L}\left(\mathcal{L}^{-1}(F)\right)=F$.

\subsection{Definitions for fractional calculus}

Since that question by L'Hôpital, different persons have tried to mathematically define fractional calculus. As far back as we can go, the first to somehow give a definition was Lacroix in his text 60]. Starting with $y=x^{m}$, where $m>0$, he showed that

$$
\frac{d^{1 / 2}}{d x^{1 / 2}} f(x)=\frac{2 \sqrt{x}}{\sqrt{\pi}}
$$

for $m=1$ and $n=\frac{1}{2}$ by generalising the $n$ th-derivative using $\Gamma$ for generalised factorial to arrive at

$$
\frac{d^{n}}{d x^{n}} f(x)=\frac{\Gamma(m+1)}{\Gamma(m-n+1)} x^{m-n} .
$$

It is generally believed that Abel was the first to apply fractional calculus by obtaining the solution of an integral equations - which was later renamed after him in his honour - that arises in the formulation of a Tautocrone problem [48. Williams [53] observed the relationship between Abel's equation and the fractional calculus using repeated integration to arrive at:

$$
\int_{a}^{x} \frac{(x-\tau)^{n-1}}{(n-1) !} f(\tau) d \tau=\int_{a}^{x} d t_{1} \int_{a}^{t_{1}} d t_{2} \cdots \int_{a}^{t_{n-1}} f\left(t_{n}\right) d t_{n}:=I_{a}^{n} f(x),
$$

where $I_{a}^{n}$ is the $n$-fold integration. Eq. 2.3 is Cauchy's integral formula which is well-defined for non-integer $n$. A resulting expression of $(2.3)$ is

$$
I_{\alpha+}^{\alpha} f(t):=\frac{1}{\Gamma(\alpha)} \int_{a}^{t}(t-\tau)^{\alpha-1} f(\tau) d \tau, t, \alpha>0
$$

which is the Riemann-Liouville fractional integral - one of the most common definitions of fractional integrals in the literature! Setting $a=0$ in 2.4 we have 
the Riemann-Liouville derivatives given by the equation

$$
{ }_{0} I_{t}^{\alpha} f(t)=\frac{1}{\Gamma(n-\alpha)} \frac{d^{n}}{d t^{n}} \int_{0}^{t}(t-\tau)^{n-\alpha-1} f(\tau) d \tau, \quad n \geq \alpha>n-1
$$

where $\Gamma$ is the Gamma function defined as

$$
\Gamma(z)=\int_{0}^{\infty} e^{-t} t^{z-1} d t, \quad \operatorname{Re}(z)>0, \quad z \in \mathbb{Z}
$$

with

$$
\Gamma(z+1)=\int_{0}^{\infty} e^{-t} t^{z} d t=-\left.e^{-t} t^{z}\right|_{t=0} ^{t=\infty}+z \int_{0}^{\infty} e^{-t} t^{z-1} d t=z \Gamma(z),
$$

and

$$
\Gamma(n+1)=n !
$$

Definition 2.7. [19] Suppose that $\alpha>0, t>0, \alpha, t \in \mathbb{R}$, the Caputo fractional derivative of order $\alpha$ is defined as

$$
D_{t}^{\alpha} f(x, t)=I^{n-\alpha} D^{n} f(x, t)= \begin{cases}\frac{1}{\Gamma(\alpha-n)} \int_{0}^{t} \frac{f^{(n)}(\tau)}{(t-\tau)^{\alpha+1-n}} d \tau & n-1<\alpha<n \in \mathbb{N} \\ \frac{d^{n}}{d t^{n}} f(t) & \alpha=n \in \mathbb{N} .\end{cases}
$$

Though they have different forms, the Caputo fractional derivative and Riemann-Liouville integral operator are related by the relations [12.

$$
\begin{aligned}
& D^{\alpha}\left(I^{\alpha} f(x, t)\right)=f(x, t) \text { and } \\
& I^{\alpha}\left(D^{\alpha} f(x, t)\right)=I^{\alpha}\left(I^{n-\alpha} f^{(n)}(x, t)\right)=I^{n} f^{(n)}(x, t)=f(x, t)-\sum_{k=0}^{n-1} f^{(k)}(x, 0) \frac{t^{k}}{k !} .
\end{aligned}
$$

The Caputo definition is a very important definition in the field of fractional calculus especially when we aim to apply it to real-life problems. Some advantages it posseses over the Riemann-Liouville definition are: under natural condition on the function $f(t)$, for $\alpha \rightarrow n$ the Caputo derivative becomes a conventional $n$th derivative of the function $f(t)$. Also, its fractional derivative of a constant is zero; when the Laplace transform of the Caputo fractional derivative is taken, its initial values are in terms of integer order derivatives (compare Lemma 2.8. Other reasons why we stick to the Caputo derivatives in this article are: 
1. Caputo's fractional differentiation, like integer-order differentiation, is a linear operation. That is,

$$
D^{\alpha}(\beta f(t)+\gamma g(t))=\beta D^{\alpha} f(t)+\gamma D^{\alpha} g(t)
$$

where $\beta$ and $\gamma$ are constants;

2. Caputo's fractional differentiation satisfies Leibniz's rule

$$
D^{\alpha}(\beta f(t) \gamma g(t))=\sum_{i=0}^{\infty}\left(\begin{array}{l}
\alpha \\
i
\end{array}\right) g^{(i)}(t) D^{\alpha-i} f(t),
$$

if $f(\tau)$ is continuous in $[0, t]$ and $g(\tau)$ has $(n+1)$ continuous derivatives in $[0, t]$.

Lemma 2.8. [4] Suppose $p>0$ and $F(s)$ is the Laplace transform of $f(t)$. The Laplace transform of the Caputo fractional derivative of order $\alpha$ is given by

$$
\mathcal{L}\left\{D_{t}^{\alpha} f(t) ; s\right\}=s^{\alpha} F(s)-\sum_{k=0}^{n-1} s^{\alpha-k-1} f^{(k)}(0), \quad n-1<\alpha<n .
$$

\section{Laplace Homotopy Analysis Method}

The LHAM, as far as we checked, was proposed by [44] and it has since been used by other researchers for different problems - see [63] for a brief list of authors. Consider the fractional differential equation

$$
D_{t}^{2 \alpha} u(t)=g\left(u(t), u_{x}(t), u_{x x}(t)\right), \quad t \geq 0, \quad 0<\alpha \leq 1
$$

with the initial conditions:

$$
u(0)=a, u_{t}(0)=b
$$

where $D_{t}^{2 \alpha}$ is Caputo's derivative. Apply the Laplace transform, denoted $\mathcal{L}$, to both sides of Eq.(3.1) and by the linearity of Laplace transforms we get

$$
\mathcal{L}\left(D_{t}^{2 \alpha} u(t)\right)=\mathcal{L}\left(g\left(u(t), u_{x}(t), u_{x x}(t)\right)\right) .
$$


Using the initial condition 3.2 , then we get

$$
s^{2 \alpha} \tilde{u}(s)-s^{2 \alpha-1} a-s^{2 \alpha-2} b=\mathcal{L}\left(g\left(u(t), u_{x}(t), u_{x x}(t)\right)\right),
$$

and consequently,

$$
\tilde{u}(s)=\frac{a}{s}+\frac{b}{s^{2}}+\frac{1}{s^{2 \alpha}} \mathcal{L}\left(g\left(u(t), u_{x}(t), u_{x x}(t)\right)\right),
$$

where $\mathcal{L}(u(t))=\tilde{u}(s)$. The zero-order deformation equation of the Laplace equation 3.3 has the form

$$
(1-q)\left[\tilde{\phi}(s ; q)-\tilde{u}_{0}(s)\right]=q \hbar\left[\tilde{\phi}(s ; q)-\frac{a}{s}-\frac{b}{s^{2}}-\frac{1}{s^{2 \alpha}} \mathcal{L}\left(g\left(u(t), u_{x}(t), u_{x x}(t)\right)\right)\right]
$$

where $q \in[0,1]$ is an embedding parameter. When $q=0$ and $q=1$, we have

$$
\tilde{\phi}(s ; 0)=\tilde{u}_{0}(s) \text { and } \tilde{\phi}(s ; 1)=\tilde{u}(s)
$$

respectively. Thus, as $q$ increases from 0 to $1, \tilde{\phi}(s ; q)$ varies from $\tilde{u}_{0}(s)$ to $\tilde{u}(s)$ and the Taylor's expansion of $\tilde{\phi}(s ; q)$

$$
\tilde{\phi}(s ; q)=\tilde{u}_{0}(s)+\sum_{m=1}^{\infty} \tilde{u}_{m}(s) q^{m},
$$

where

$$
\tilde{u}_{m}(s)=\left.\frac{1}{m !} \frac{\partial^{m} \tilde{\phi}(s ; q)}{\partial q^{m}}\right|_{q=0} .
$$

If the auxiliary parameter $\hbar$ and the initial guesses $\tilde{u}_{0}(s)$ are properly chosen, then (3.5) converges at $q=1$ and we have

$$
\tilde{u}(s)=\tilde{u}_{0}(s)+\sum_{m=1}^{\infty} \tilde{u}_{m}(s) .
$$

Define the vector

$$
\overrightarrow{\tilde{u}}_{m}(s)=\left\{\tilde{u}_{0}(s), \tilde{u}_{1}(s), \ldots, \tilde{u}_{m}(s)\right\} .
$$


Differentiating (3.4) $m$ times with respect to $q$ [by using the Leibniz rule defined in [2.10] ], setting $q=0, \hbar=-1$ and finally dividing through by $m$ !, we have the $m$ th-order deformation equation

$$
\tilde{u}_{m}(s)=\chi_{m} \tilde{u}_{m-1}(s)-R_{m}\left(\overrightarrow{\tilde{u}}_{m-1}(s)\right),
$$

where

$$
\begin{aligned}
R_{m}\left(\overrightarrow{\vec{u}}_{m-1}(s)\right) & =\tilde{u}_{m-1}(s)-\left(\frac{a}{s}+\frac{b}{s^{2}}\right)\left(1-\chi_{m}\right) \\
& -\frac{1}{s^{2 \alpha}}\left(\frac{1}{(m-1) !} \frac{\partial^{m-1}}{\partial q^{m-1}}\left[\mathcal{L}\left(g\left(t, \phi(t ; q), \frac{d}{d t} \phi(t, q)\right)\right)\right]_{q=0}\right)
\end{aligned}
$$

and

$$
\chi_{m}= \begin{cases}0, & \text { if } m \leq 1 \\ 1, & \text { if } m>1\end{cases}
$$

Taking the inverse Laplace transform, that is $\mathcal{L}^{-1}$, of 3.6 gives a power series solution

$$
u(t)=\sum_{i=0}^{\infty} u_{i}(t) .
$$

\subsection{Convergence of LHAM}

Theorem 3.1. Suppose $\tilde{\phi}(s ; q)=\tilde{u}_{0}(s)+\sum_{m=1}^{\infty} \tilde{u}_{m}(s) q^{m}$ [from Eq. 3.5)]. Then the solution of (3.4) resulting from (3.1) exists as power series in $q$, the embedding parameter, if $\lim _{m \rightarrow \infty} \tilde{\phi}_{m}(s)=\tilde{\phi}(s)$.

Proof. See [36].

Theorem 3.2 (Convergence theorem). As long as the series (3.8) is convergent, where $u_{i}(t)$ is produced by the mth-order deformation equation (3.6). It must be the exact solution of (3.1).

Proof. Suppose $\sum_{i=0}^{\infty} u_{i} t$ converges uniformly to $u(t)$, then

$$
\lim _{i \rightarrow \infty} u_{i}(t)=0 \text { for all } t \in \mathbb{R}^{+} .
$$


Since $\mathcal{L}^{-1}$, like its corresponding Laplace transform, is a linear operator, we have:

$$
\begin{aligned}
\sum_{m=1}^{i} \mathcal{L}^{-1}\left[\tilde{u}_{m}(s)-\chi_{m} \tilde{u}_{m-1}(s)\right] & =\sum_{m=1}^{i}\left[\mathcal{L}^{-1} \tilde{u}_{m}(s)-\chi_{m} \mathcal{L}^{-1} \tilde{u}_{m-1}(s)\right] \\
& =\mathcal{L}^{-1} \tilde{u}_{1}(s)+\cdots+\left[\mathcal{L}^{-1} \tilde{u}_{i}(s)-\mathcal{L}^{-1} \tilde{u}_{i-1}(s)\right] \\
& =\mathcal{L}^{-1} \tilde{u}_{i}(s) \\
& =u_{i}(t)
\end{aligned}
$$

From (3.6), 3.9) and (3.10), we have:

$$
\begin{aligned}
\sum_{m=1}^{\infty} \mathcal{L}^{-1}\left[\tilde{u}_{m}(s)-\chi_{m} \tilde{u}_{m-1}(s)\right] & =\sum_{m=1}^{\infty}\left[u_{m}(t)-\chi_{m} u_{m-1}(t)\right] \\
& =\lim _{i \rightarrow \infty} u_{i}(t)=0
\end{aligned}
$$

hence

$$
\hbar \sum_{m=1}^{\infty} R_{m}\left(\tilde{u}_{m-1}\right)=\sum_{m=1}^{\infty}\left[\tilde{u}_{m}(s)-\chi_{m} \tilde{u}_{m-1}(s)\right]=0
$$

since $\hbar \neq 0$, this yields

$$
\sum_{m=1}^{\infty} R_{m}\left(\tilde{u}_{m-1}\right)=0 .
$$

Now from Theorem 2.3 and (3.7),

$$
\begin{aligned}
0 & =\sum_{m=1}^{\infty}\left[\tilde{u}_{m-1}(s)-\frac{1}{s^{2 \alpha}} \mathcal{L}\left(g\left(u(t), u_{x}(t), u_{x x}(t)\right)\right)-\left(\frac{a}{s}+\frac{b}{s^{2}}\right)\left(1-\chi_{m}\right)\right] \\
& =\sum_{m=1}^{\infty} \tilde{u}_{m-1}(s)-\sum_{m=1}^{\infty} \frac{1}{s^{2 \alpha}} \mathcal{L}\left(g\left(u(t), u_{x}(t), u_{x x}(t)\right)\right)-\sum_{m=1}^{\infty}\left(\frac{a}{s}+\frac{b}{s^{2}}\right)\left(1-\chi_{m}\right) \\
& =\tilde{u}(s)-\left(\frac{a}{s}+\frac{b}{s^{2}}\right)-\frac{1}{s^{2 \alpha}} \mathcal{L}\left(g\left(u(t), u_{x}(t), u_{x x}(t)\right)\right)
\end{aligned}
$$

hence,

$$
\tilde{u}(s)=\frac{a}{s}+\frac{b}{s^{2}}+\frac{1}{s^{2 \alpha}} \mathcal{L}\left(g\left(u(t), u_{x}(t), u_{x x}(t)\right)\right)
$$

and

$$
\tilde{u}(s)-\frac{a}{s}-\frac{b}{s^{2}}=\frac{1}{s^{2 \alpha}} \mathcal{L}\left(g\left(u(t), u_{x}(t), u_{x x}(t)\right)\right)
$$


which implies that

$$
s^{2 \alpha} \tilde{u}(s)-s^{2 \alpha-1} a-s^{2 \alpha-2} b=\mathcal{L}\left(g\left(u(t), u_{x}(t), u_{x x}(t)\right)\right) .
$$

Since $a=u(0)$ and $b=u_{t}(0)$ from (3.2), 3.16) becomes

$$
s^{2 \alpha} \tilde{u}(s)-s^{2 \alpha-1} u(0)-s^{2 \alpha-2} u_{t}(0)=\mathcal{L}\left(g\left(u(t), u_{x}(t), u_{x x}(t)\right)\right) .
$$

The left hand side of (3.17) takes the form of 2.11 which tells us that 3.17) is the Laplace transform of a derivative which in our case is a Caputo fractional derivative. Thus, we have

$$
\mathcal{L}\left(D_{t}^{2 \alpha} u(t)\right)=\mathcal{L}\left(g\left(u(t), u_{x}(t), u_{x x}(t)\right)\right) .
$$

Taking the inverse Laplace transform of both sides, we have:

$$
D_{t}^{2 \alpha} u(t)=g\left(u(t), u_{x}(t), u_{x x}(t)\right) .
$$

Therefore, $u(t)$ is the exact solution and the proof is complete.

\section{Application}

In this section, we apply the LHAM to some equations.

\subsection{The fractional cubic Boussinesq equation}

The cubic Boussinesq equation of fractional order is the equation given as

$$
\begin{array}{r}
D_{t}^{2 \alpha} u(x, t)-u_{x x}(x, t)+2\left(u^{3}\right)_{x x}(x, t)-(u)_{x x x x}(x, t)=0, \\
u(x, 0)=\frac{1}{x}, u_{t}(x, 0)=\frac{-1}{x^{2}}, 0<\alpha \leq 1 .
\end{array}
$$

The $m$ th-order deformation equation is thus given as

$$
\begin{aligned}
& u_{m}(x, s)=\chi_{m} u_{m-1}(x, s)-u_{m-1}(x, s)+\left(\frac{1}{s x}-\frac{1}{(s x)^{2}}\right)\left(1-\chi_{m}\right) \\
& +\mathcal{L}\left(\frac{1}{s^{2 \alpha}}\left(u_{m-1}\right)_{x x}(x, t)-\frac{2}{s^{2 \alpha}}\left(\sum_{j=0}^{m-1} u_{m-1-j} \sum_{i=0}^{j} u_{i} u_{j-1}\right)_{x x}+\frac{1}{s^{2 \alpha}}\left(u_{m-1}\right)_{x x x x}\right)
\end{aligned}
$$


and

$$
\chi_{m}=\left\{\begin{array}{ll}
0, & \text { if } m \leq 1 \\
1, & \text { if } m>1
\end{array} .\right.
$$

Therefore,

$$
\begin{gathered}
u_{0}(x, s)=\frac{1}{s x}-\frac{1}{(s x)^{2}} \\
u_{1}(x, s)=\frac{1}{s^{2 \alpha}}\left[\left(\frac{2 !}{s x^{3}}-\frac{3 !}{s^{2} x^{4}}\right)+\ldots\right]=\frac{1}{s^{2 \alpha+1}} \frac{2 !}{x^{3}}-\frac{1}{s^{2 \alpha+2}} \frac{3 !}{x^{4}}+\ldots
\end{gathered}
$$

Hence

$$
u(x, s)=\frac{1}{s x}-\frac{1}{s^{2} x^{2}}+\frac{1}{s^{2 \alpha+1}} \frac{2 !}{x^{3}}-\frac{1}{s^{2 \alpha+2}} \frac{3 !}{x^{4}}+-\cdots
$$

Taking the inverse Laplace transform, we have

$$
u(x, t)=\frac{1}{x}-\frac{t}{x^{2}}+\frac{2 !}{x^{3}} \frac{t^{2 \alpha}}{\Gamma(2 \alpha+1)}-\frac{3 !}{x^{4}} \frac{t^{3 \alpha}}{\Gamma(2 \alpha+2)}+-\cdots
$$

For a test case, let $\alpha=1$, then we have

$$
u(x, t)=\frac{1}{x}-\frac{t}{x^{2}}+\frac{t^{2}}{x^{3}}-\frac{t^{3}}{x^{4}}+\cdots=\frac{1}{x} \sum_{n=0}^{\infty}(-1)^{n}\left(\frac{t}{x}\right)^{n}=\frac{1}{x+t}
$$

which coincides with the exact solution. See [5].

\subsection{The fractional Boussinesq-like $B(n, n)$ equation}

The Boussinesq-like $B(n, n)$ equation is the equation given as

$$
D_{t}^{2 \alpha} u(x, t)-\left(u^{n}\right)_{x x}(x, t)-\left(u^{n}\right)_{x x x x}(x, t)=0, m, n>1 .
$$

For this particular equation, we would like to choose two cases, namely, $B(2,2)$ and $B(3,3)$ with distinct initial conditions to illustrate the efficiency of LHAM.

Case 1. When $n=2$, 4.4 with initial condition is given as

$$
D_{t}^{2 \alpha} u(x, t)-\left(u^{2}\right)_{x x}(x, t)-\left(u^{2}\right)_{x x x x}(x, t)=0,
$$




$$
u(x, 0)=\frac{4}{3} a^{2} \sin ^{2}\left(\frac{x}{4}\right), u_{t}(x, 0)=\frac{a^{3}}{3} \sin \left(\frac{x}{2}\right),
$$

where $a$ is an arbitrary constant. Based on the Section 3 , the $m$-th order deformation equation of (4.5) using (4.6) is given by the equation

$$
\begin{aligned}
u_{m}(x, s)= & \chi_{m} u_{m-1}(x, s)-u_{m-1}(x, s) \\
& +\left[\frac{1}{s}\left(\frac{4}{3} a^{2} \sin ^{2}\left(\frac{x}{4}\right)\right)+\frac{1}{s^{2}}\left(\frac{a^{3}}{3} \sin \left(\frac{x}{2}\right)\right)\right]\left(1-\chi_{m}\right) \\
& +\mathcal{L}\left(\frac{1}{s^{2 \alpha}} \sum_{j=0}^{m-1}\left(u_{j} u_{m-1-j}\right)_{x x}(x, t)+\frac{1}{s^{2 \alpha}} \sum_{j=0}^{m-1}\left(u_{j} u_{m-1-j}\right)_{x x x x}(x, t)\right)
\end{aligned}
$$

where

$$
\chi_{m}= \begin{cases}0, & \text { if } m \leq 1 \\ 1, & \text { if } m>1\end{cases}
$$

which, when $m \geq 1$, is given by the equation

$$
u_{m}(x, s)=\mathcal{L}\left(\frac{1}{s^{2 \alpha}} \sum_{j=0}^{m-1}\left(u_{j} u_{m-1-j}\right)_{x x}(x, t)+\frac{1}{s^{2 \alpha}} \sum_{j=0}^{m-1}\left(u_{j} u_{m-1-j}\right)_{x x x x}(x, t)\right) .
$$

From (4.7),

$$
u_{0}(x, s)=\frac{1}{s} \frac{4}{3} a^{2} \sin ^{2}\left(\frac{x}{4}\right)+\frac{1}{s^{2}} \frac{a^{3}}{3} \sin \left(\frac{x}{2}\right)
$$

which implies, by inverse Laplace transform, that

$$
u_{0}(x, t)=\frac{4}{3} a^{2} \sin ^{2}\left(\frac{x}{4}\right)+\frac{a^{3}}{3} t \sin \left(\frac{x}{2}\right)
$$

Inverting (4.8) using Laplace transform, we have

$$
u_{m}(x, t)=\mathcal{L}^{-1}\left(\frac{1}{s^{2 \alpha}} \sum_{j=0}^{m-1}\left(u_{j} u_{m-1-j}\right)_{x x}(x, t)+\frac{1}{s^{2 \alpha}} \sum_{j=0}^{m-1}\left(u_{j} u_{m-1-j}\right)_{x x x x}(x, t)\right) .
$$


By repeating this procedure for $u_{1}, u_{2} \ldots$ and setting $\alpha=1$ we have the series solution

$$
u(x, t)=\frac{4}{3} a^{2} \sin ^{2}\left(\frac{x}{4}\right)+\frac{a^{3}}{3} t \sin \left(\frac{x}{2}\right)+\frac{a^{4}}{12} t^{2} \cos \left(\frac{x}{2}\right)-\frac{a^{5}}{72} t^{3} \sin \left(\frac{x}{2}\right)+\ldots
$$

Using Taylor series we get

$$
u(x, t)=\frac{4}{3} a^{2} \sin ^{2}\left(\frac{x+a t}{4}\right),-2 \pi \leq x+a t \leq 2 \pi
$$

which coincides with the exact solution. See [72].

Case 2. When $n=3$, (4.4) with initial condition is given as

$$
D_{t}^{2 \alpha} u(x, t)-\left(u^{3}\right)_{x x}(x, t)-\left(u^{3}\right)_{x x x x}(x, t)=0,
$$

$u(x, 0)=\frac{\sqrt{6}}{2} a b \sin \left(\frac{x}{3}\right)+\frac{\sqrt{6}}{2} a b \cos \left(\frac{x}{3}\right), u_{t}(x, 0)=\frac{\sqrt{6}}{2} a b^{2} \cos \left(\frac{x}{3}\right)-\frac{\sqrt{6}}{2} a b^{2} \sin \left(\frac{x}{3}\right)$,

where $a$ and $b$ are arbitrary constants. The $m$-th order deformation equation of (4.14) is the equation

$$
\begin{aligned}
& u_{m}(x, s)=\chi_{m} u_{m-1}(x, s)-u_{m-1}(x, s) \\
& +\left[\frac{1}{s}\left(\frac{\sqrt{6}}{2} a b \sin \left(\frac{x}{3}\right)+\frac{\sqrt{6}}{2} a b \cos \left(\frac{x}{3}\right)\right)+\frac{1}{s^{2}}\left(\frac{\sqrt{6}}{2} a b^{2} \cos \left(\frac{x}{3}\right)-\frac{\sqrt{6}}{2} a b^{2} \sin \left(\frac{x}{3}\right)\right)\right]\left(1-\chi_{m}\right) \\
& +\frac{1}{s^{2 \alpha}} \mathcal{L}\left[\left(\sum_{i=0}^{m-1} u_{m-1-i} \sum_{j=0}^{i} u_{j} u_{i-j}\right)_{x x}(x, t)+\left(\sum_{i=0}^{m-1} u_{m-1-i} \sum_{j=0}^{i} u_{j} u_{i-j}\right)_{x x x x}(x, t)\right],
\end{aligned}
$$

where

$$
\chi_{m}=\left\{\begin{array}{ll}
0, & \text { if } m \leq 1 \\
1, & \text { if } m>1
\end{array} .\right.
$$

From 4.16,

$$
u_{0}(x, s)=\frac{1}{s}\left(\frac{\sqrt{6}}{2} a b \sin \left(\frac{x}{3}\right)+\frac{\sqrt{6}}{2} a b \cos \left(\frac{x}{3}\right)\right)+\frac{1}{s^{2}}\left(\frac{\sqrt{6}}{2} a b^{2} \cos \left(\frac{x}{3}\right)-\frac{\sqrt{6}}{2} a b^{2} \sin \left(\frac{x}{3}\right)\right) .
$$


Inverse Laplace transformation of (4.17) gives us

$$
\begin{aligned}
u_{0}(x, t) & =\frac{\sqrt{6}}{2} a b \sin \left(\frac{x}{3}\right)+\frac{\sqrt{6}}{2} a b \cos \left(\frac{x}{3}\right)+t\left(\frac{\sqrt{6}}{2} a b^{2} \cos \left(\frac{x}{3}\right)-\frac{\sqrt{6}}{2} a b^{2} \sin \left(\frac{x}{3}\right)\right) \\
& =\frac{\sqrt{6}}{2} a b\left[(1-b t) \sin \left(\frac{x}{3}\right)+(1+b t) \cos \left(\frac{x}{3}\right)\right] .
\end{aligned}
$$

Using the inverse (Laplace transform) of 4.16 with $u_{0}(x, t)$ recursively for values of $u_{1}, u_{2} \ldots$ we get a series solution of 4.14. Upon setting $\alpha=1$ and using Taylor series we get

$$
u(x, t)=\frac{\sqrt{6}}{2} a b \sin \left(\frac{x+b t}{3}\right)+\frac{\sqrt{6}}{2} a b \cos \left(\frac{x+b t}{3}\right)
$$

which coincides with the exact solution. See [39]

\section{Discussion of Results}

We have solved for the closed form solution of generalised Boussinesq equations of fractional order using a simple and concise approach allowed by the Laplace Homotopy Analysis Method. Results obtained are for values of $\alpha=1$. Other values of $0<\alpha<1$ can also be found by extension.

\section{Conflicts of Interest}

Authors declare no conflicting interest.

\section{Acknowledgement}

The first author will like to thank Drs. Lanre Akinyemi and Mehmet Şenol of Prairie View A\&M University and Nevşehir Haci Bektaş University respectively for their comments and valuable insights. 


\section{References}

[1] A. Aghili and J. Aghili, Exponential differential operators for singular integral equations and space fractional Fokker-Planck equation, Bol. Soc. Parana. Mat. 36 (2018), 223-233. https://doi.org/10.5269/bspm.v36i1.30959

[2] A. Carpinteri and F. Mainardi, Fractals and Fractional Calculus in Continuum Mechanics, Springer Verlag, Vienna, New York, 1997. https://doi .org/10.1007/978-3-7091-2664-6

[3] A. Shirzadi, L. Ling and S. Abbasbandy, Meshless simulations of the two dimensional fractional-time convection-diffusion-reaction equations, Eng. Anal. Bound. Elem. 38 (2012), 1522-1527. https://doi.org/10.1016/j.enganabound.2012.05.005

[4] A.A. Kilbas, H.M. Srivastava and J.J. Trujillo, Theory and Application of Fractional Differential Equations, Elseiver, 2006.

[5] A.M. Wazwaz, The variational iteration method for rational solutions for $\mathrm{KdV}$, $K(2,2)$, Burgers and cubic Boussinesq equations, Journal of Computational and Applied Math. 207 (2007), 18-23. https://doi.org/10.1016/j.cam.2006.07.010

[6] C.A. Monje, Y. Chen, B.M. Vinagre, D. Xue and V. Feliu-Batile, Fractional-order Systems and Controls: Fundamentals and Applications, Springer-Verlag London Ltd., 2010. https://doi.org/10.1007/978-1-84996-335-0

[7] C. Chen, F. Liu and K. Burrage, Finite difference methods and a fourier analysis for the fractional reaction-subdiffusion equation, Appl. Math. Comput. 198 (2008), 754-769. https://doi.org/10.1016/j.amc.2007.09.020

[8] D. Kaya, Explicit solutions of generalized Boussinesq equations, J. Appl. Math. 1 (2001), 29-37. https://doi.org/10.1155/S1110757X01000067

[9] D.E. Rupp and J.S. Selker, On the use of the Boussinesq equation for interpreting recession hydrographs from sloping aquifers, Water Resour. Res. 42 (2006), W12421. https://doi.org/10.1029/2006WR005080

[10] E. Ahmed, A.M.A. El-Sayed and H.A.A. El-Saka, Equilibrium points, stability and numerical solutions of fractional-order predator-prey and rabies models, J. Math. Anal. Appl. 325 (2007), 542-553. https://doi.org/10.1016/j.jmaa.2006.01.087 
[11] F. Mainardi, Fractional calculus and waves in linear viscoelasticity, An Introduction to Mathematical Models, Imperial College Press, 2010.

https://doi.org/10.1142/p614

[12] F. Özpinar, Applying discrete homotopy analysis method for solving fractional partial differential equations, Entropy 20 (2018), 332.

https://doi.org/10.3390/e20050332

[13] G. Adomian, A review of the decomposition method in applied mathematics, $J$. Math. Anal. Appl. 135 (1988), 501-544.

https://doi.org/10.1016/0022-247X(88)90170-9

[14] G. Adomian, Solving Frontier Problems in Physics: The Decomposition Method, Kluwer, Boston, 1994. https://doi.org/10.1007/978-94-015-8289-6

[15] G. Wei, J.T. Kirby, S.T. Grilli and R. Subramanya, A fully nonlinear Boussinesq model for surface waves. Part 1. Highly nonlinear unsteady waves, J. Fluid Mech. 294 (1995), 71-92. https://doi.org/10.1017/S0022112095002813

[16] G. Wei and J.T. Kirby, Time-dependent numerical code for extended Boussinesq equations, J. Waterway, Port, Coastal, and Ocean Eng. 121(5) (1995), 251-261. https://doi.org/10.1061/(ASCE)0733-950X(1995)121:5(251)

[17] H. Richard, It's a Nonlinear World, Springer, New York, NY, USA, 2011.

[18] I. Petráš, Fractional derivatives, fractional integrals and fractional differential equations in Matlab, Engineering Education and Research using Matlab, InTech, 2011.

[19] I. Podlubny, Fractional Differential Equations, Academic Press, New York, 1999.

[20] J. Boussinesq, Théorie de l'intumescence appelée onde solitaire ou de translation se propagente dans un canal rectangulaire, Comptes Rendus 72 (1871), 755-759.

[21] J. Boussinesq, Théorie des ondes et des remous qui se propagent le long d'un canal rectangulaire horizontal, en communiquant au liquide contenu dans ce canal des vitesses sensiblemant parielles de la surface au fond, J. Pure Appl. 7 (1872), 55-108.

[22] J. Boussinesq, Essai sur la théorie des eaux courantes, Mem. Acad. Sci. Inst. Fr. 23 (1877), 252-260. 
[23] J. Boussinesq, Sur le débit, en temps de sécheresse, d'une source alimentée par une nappe d'eaux d'infiltration, C.R. Hebd. Seanc. Acad. Sci. Paris 136 (1903), 1511-1517.

[24] J. Boussinesq, Recherches théoriques sur l'écoulement des nappes d'eau infiltrées dans le sol et sur débit de sources, J. Math. Pures Appl. 10 (1904), 5-78.

[25] J.A.T. Machado, A probabilistic interpretation of the fractional-order differentiation, Frac. Calc. Appl. 6 (2003), 73-80.

[26] J.H. He, Homotopy perturbation technique, Comp. Meth. Appl. Mech. Engnr. 178(3-4) (1999), 256-262. https://doi.org/10.1016/S0045-7825(99)00018-3

[27] J.H. He, Variational iteration method - a kind of nonlinear analytical technique: some examples, Int. J. Nonlin. Mech. 34(4) (1999), 699-708.

https://doi.org/10.1016/S0020-7462(98)00048-1

[28] J.H. He, Variational iteration method. Some recent results and new interpretations, J. Comput. Appl. Math. 207 (2007), 3-17. https://doi.org/10.1016/j.cam.2006.07.009

[29] J.P. Roop, Computational aspects of FEM approximations of fractional advection dispersion equations on bounded domain in $\mathbb{R}^{2}$, J. Comp. Appl. Math. 193 (2006), 243-268. https://doi.org/10.1016/j.cam.2005.06.005

[30] J.S. Russell, Report on waves, Proceedings of the 14th Meeting of the British Association for the Advancement of Science, 1884.

[31] K. Al-Khaled and S. Momani, An approximate solution for a fractional diffusion-wave equation using the decomposition method, Appl. Math. Comp. 165 (2005), 473-483. https://doi.org/10.1016/j.amc.2004.06.026

[32] K. Diethelm, The Analysis of Fractional Differential Equations, Springer, New York, NY, USA, 2010. https://doi.org/10.1007/978-3-642-14574-2

[33] K.B. Oldham and J. Spanier, The Fractional Calculus, Academic Press, New York, USA, 1974.

[34] K.S. Miller and B. Ross, An Introduction to the Fractional Calculus and Fractional Differential Equations, Wiley, New York, 1993. 
[35] K.V. Zhukovsky, The operational solution of fractional-order differential equations as well as Black-Scholes and heat-conduction equations, Mosc. Uni. Phys. Bull. 71 (2016), 237-244. https://doi.org/10.3103/S0027134916030164

[36] L. Akinyemi and S.N. Huseen, A powerful approach to study the new modified coupled Korteweg-de Vries system, Math. Comp. Sim. 177 (2020), 556-567. https://doi.org/10.1016/j.matcom.2020.05.021

[37] L. Moslehi and A. Ansari, On M-Wright transforms and time-fractional diffusion equations, Integral transforms Spec. Funct. 28 (2017), 113-129.

https://doi.org/10.1080/10652469.2016.1252763

[38] L. Su, W. Wang and Q. Xu, Finite difference methods for fractional dispersion equations, Appl. Math. Comput. 216 (2010), 3329-3334.

https://doi.org/10.1016/j.amc.2010.04.060

[39] M. Deghan, J. Manafian and A. Saadatmandi, Solving Nonlinear Fractional Partial Differential Equations using the Homotopy Analysis Method, Wiley InterScience, 2009. https://doi.org/10.1002/num.20460

[40] M. Deghan, M. Abbaszadeh and A. Mohebbi, An implicit RBF meshless approach for solving time fractional nonlinear Sine-Gordon and Klein-Gordon equation, Eng. Anal. Bound. Elem. 50 (2015), 412-434.

https://doi.org/10.1016/j.enganabound.2014.09.008

[41] M. Diethelm and A.D. Freed, On the solution of nonlinear fractional order differential equations used in modelling of viscoplasticity, Scientific Computing in Chemical Engineering and Computational Fluid Dynamics, Reaction Engineering and Molecular Properties, Springer, Heidelberg, 1999, pp. 217-224.

https://doi.org/10.1007/978-3-642-60185-9_24

[42] M. Eslami, B.F. Vajargah, M. Mirzazadeh and A. Biswas, Applications of first integral method to fractional partial differential equations, Indian J. Phys. 88 (2014), 177-184. https://doi.org/10.1007/s12648-013-0401-6

[43] M. Sajid and T. Hayat, Comparison of HAM and HPM methods in nonlinear heat conduction and convection equations, Nonlinear Anal. Real World Appl. 9 (2008), 2296-2301. https://doi.org/10.1016/j.nonrwa.2007.08.007 
[44] M. Zurigat, Solving fractional oscillators using Laplace homotopy analysis method, Annals of the University of Craiova, Mathematics and Computer Science Series 38(4) (2011), 1-11.

[45] M.F. Gobbi, J.T. Kirby and G. Wei, A fully nonlinear Boussinesq model for surface waves. Part 2. Extension to $O(k h)^{4}$, J. Fluid Mech. 405 (2000), 181-210.

https://doi.org/10.1017/S0022112099007247

[46] M.J. Ablowitz, Nonlinear dispersive waves, Asymptotic Analysis and Solitons, Cambridge Univ. Press, 2011.

[47] M.S.H. Chowdhury, I. Hashim and O. Abdulaziz, Comparison of homotopy analysis method and homotopy perturbation method for purely nonlinear fin-type problems, Commun. Nonlinear Sci. Numeri. Simul. 14 (2009), 371-378.

https://doi.org/10.1016/j.cnsns.2007.09.005

[48] N. Abel, Solution de quelques problémes á laide dintégrales définites, Christiania Grondhal, Norway, 1881, pp. 16-18.

[49] N.J. Ford and A.C. Simpson, Numerical approaches to the solution of some fractional differential equations, Numerical Analysis Report. Manchester Centre for Computational Mathematics, Manchester, 2003.

[50] N.J. Ford and A.C. Simpson, The numerical solution of fractional differential equations, Numerical Analysis Report, Manchester Centre for Computational Mathematics, Manchester, 2003.

[51] N.T. Shawagfeh, Analytical approximate solutions for nonlinear fractional differential equations, Appl. Math. Comput. 131(2) (2002), 517-529. https://doi.org/10.1016/S0096-3003(01)00167-9

[52] O. Nwogu, An alternative form of the Boussinesq equations for nearoffshore wave propagation, J. Waterway. Port. Coast. Ocean Eng. 119 (1993), 618-638.

https://doi.org/10.1061/(ASCE)0733-950X(1993)119:6(618)

[53] P. A. Williams, Unifying Fractional Calculus with Time Scales, PhD thesis, University of Melbourne, 2012.

[54] P. Rosenau and J.M. Hyman, Compactons: Solitons with finite wavelengths, Physical Reviews Letters, American Physical Society 70 (1993), 564-567.

https://doi.org/10.1103/PhysRevLett.70.564 
[55] P. Rosenau, "What is a compacton?", Notices of the American Mathematical Society (2005), 738-739.

[56] R. Abazari and A. Kiliçman, Solitary wave solutions of the Boussinesq equation and its improved form, Math. Probl. Eng., vol. 2013, Article ID 468206. https://doi.org/10.1155/2013/468206

[57] R. Caponetto, G. Dongola, L. Fortuna and I. Petráš, Fractional Order Systems: Modelling and Control Applications, World Scientific, River Edge, NJ, 2010. https://doi.org/10.1142/7709

[58] R. Sahadevan and T. Bakkyaraj, Invariant analysis of time fractional generalized Burgers and Korteweg-de Vries equations, J. Math. Anal. Appl. 2 (2012), 341-347. https://doi.org/10.1016/j.jmaa.2012.04.006

[59] R.L. Magin, Fractional Calculus in Bioengineering, Begell House, 2006.

[60] S. Lacroix, Traité du calcul differentiel et du calcul intégral, Courcier, Paris.

[61] S. Momani and Z. Odibat, Homotopy perturbation method for nonlinear partial differential equations of fractional order, Physics Letters A 365(5) (2007), 345-350. https://doi.org/10.1016/j.physleta.2007.01.046

[62] S.J Liao, The proposed homotopy analysis technique for the solution of nonlinear problems, PhD thesis, Shanghai Jiao Tong University, 1992.

[63] S.O. Ajibola, A.S. Oke and W.N. Mutuku, LHAM approach to fractional order Rosenau-Hyman and Burgers' equations, Asian Research Journal of Mathematics 16(6) (2020), 1-14. https://doi.org/10.9734/arjom/2020/v16i630192

[64] S.S. Ray and R.K. Bera, An approximate solution of a nonlinear fractional differential equation by Adomian decomposition method, Appl. Math. Comp. 167 (2005), 561-571. https://doi.org/10.1016/j.amc.2004.07.020

[65] S.T. Momani and N.T. Shawagfeh, Decomposition method for solving fractional Riccati differential equations, Appl. Math. Comput. 182 (2006), 1083-1092. https://doi.org/10.1016/j.amc.2006.05.008

[66] T. Hayat, S. Nadeem and S. Asghar, Periodic unidirectional flows of a viscoelastic fluid with the fractional Maxwell model, Appl. Math. Comput. 151 (2004), 153-161. https://doi.org/10.1016/S0096-3003(03)00329-1 
[67] T.J. Priestly and P.A. Clarkson, Symmetries of a generalized Boussinesq equation, IMS Technical Report, UKC/IMS/59, 1996.

[68] V. Daftardar-Gejji and H. Jafari, Adomian decomposition: A tool for solving a system of fractional differential equations, J. Math. Anal. Appl. 301(2) (2005), 508-518. https://doi.org/10.1016/j.jmaa.2004.07.039

[69] W. Deng, Finite element method for the space and time fractional Fokker-Planck equation, SIAM Journal on Numerical Analysis 47 (2008), 204-226. https://doi.org/10.1137/080714130

[70] Y. Lin and C. Xu, Finite difference/spectral approximations for the time fractional diffusion equation, J. Comput. Phys. 225(2) (2007), 1533-1552. https://doi.org/10.1016/j.jcp.2007.02.001

[71] Y. Pandir and H. Huseyin Duzgun, New exact solutions of time fractional Gardner equation by using new version of F-Expansion method, Commun. Theor. Phys. 67 (2017), 9-14. https://doi.org/10.1088/0253-6102/67/1/9

[72] Y. Zhu, Q. Chang and S. Wu, Exact solitary solutions with compact support for the nonlinear dispersive Boussinesq-like $B(m, n)$ equations, Chaos Solitons and Fractals 26 (2005), 407-413. https://doi.org/10.1016/j.chaos.2005.01.005

[73] Z. Odibat and S. Momani, Application of variation iteration method to nonlinear differential equations of fractional order, Int. J. Nonlin. Sci. Numer. Simul. 1(7) (2006), 15-27. https://doi.org/10.1515/IJNSNS.2006.7.1.27

This is an open access article distributed under the terms of the Creative Commons Attribution License (http://creativecommons.org/licenses/by/4.0/), which permits unrestricted, use, distribution and reproduction in any medium, or format for any purpose, even commercially provided the work is properly cited. 